\title{
Speeding Up Approximation Algorithms for NP-hard Spanning Forest Problems by Multi-objective Optimization
}

\author{
Frank Neumann * and Marco Laumanns *夫
}

\begin{abstract}
We give faster approximation algorithms for the generalization of two NP-hard spanning tree problems. First, we investigate the problem of minimizing the degree of minimum spanning forests. Fischer [4] has shown how to compute a minimum spanning tree of degree at most $b \cdot \Delta^{*}+\left\lceil\log _{b} n\right\rceil$ in time $O\left(n^{4+1 / \ln b}\right)$ for any $b>1$, where $\Delta^{*}$ is the value of an optimal solution. We model our generalization as a multi-objective optimization problem and give a deterministic algorithm that computes for each number of connected components a solution with the same approximation quality as the algorithm of Fischer and runs in time $O\left(n^{3+1 / \ln b}\right)$. After that, we take a multi-objective view on the problem of computing minimum spanning trees with nonuniform degree bounds, which has been examined by Könemann and Ravi [10]. Given degree bounds $B_{v}$ for each vertex $v \in V$, we construct an algorithm that computes for each number of connected components a spanning forest in which each vertex $v$ has degree $O\left(B_{v}+\log n\right)$ and whose weight is at most a constant times the weight of a minimum spanning forest obeying the degree bounds. The total runtime of our algorithm is $O\left(n^{3+2 / \ln b}\right)$ for an arbitrary constant $b>1$. Setting $b=e^{k}, k>2 / 3$ an arbitrary constant, the runtime is by a factor $n^{3-2 / k} \log n$ less than the given bound by Könemann and Ravi.
\end{abstract}

\section{Introduction}

In this paper we consider two NP-hard spanning forest problems. Given a connected graph $G=(V, E)$ with $n$ vertices and $m$ edges and positive integer weights $w(e)$ for each edge $e \in E$, we are searching (i) for minimum spanning forests of minimum degree, and (ii) for minimum spanning forests obeying given degree bounds on the vertices. A forest with $i$ connected components is a cycle-free subgraph of $G$ that contains exactly $n-i$ edges. A minimum spanning forest with $i$ connected components is a forest where the sum over all edge weights is minimal among all spanning forests with $i$ connected components. In a minimum spanning forest of minimum degree, the largest vertex degree is as small

\footnotetext{
* Institut of Computer Science, Christian-Albrechts-Univ. of Kiel, 24098 Kiel, Germany, email: fne@informatik.uni-kiel.de

** Institute of Operations Research, ETH Zürich, CH-8092 Zürich, Switzerland, email: laumanns@ifor.math.ethz.ch
} 
as possible. This generalizes the problem of computing minimum spanning trees of minimum degree.

The problem of computing minimum spanning trees is one of the fundamental problems in computer science and can be solved in polynomial time by greedy algorithms. The well-known algorithms due to Kruskal and Prim have worst-case runtimes of $O((n+m) \log n)$ and $O\left(n^{2}\right)$, respectively (see, e.g., Cormen, Leiserson, Rivest, and Stein [3]). The problem of constructing spanning subgraphs that satisfy given constraints has attained a lot of attention (see, e.g., [1,2]). In most cases, even simple constraints lead to NP-hard problems (see Garey and Johnson [8]). The problem of minimizing the degree of spanning trees is NP-hard as a spanning tree of degree 2 is a Hamiltonian path. Constrained spanning tree problems have a lot of application in the area of network design.

\subsection{Previous work and our results}

Let $\Delta^{*}$ be the degree of an optimal solution. When edge weights are not considered, or assumed uniform, a $\Delta^{*}+1$ approximation algorithm for minimizing the degree of spanning trees has been obtained by Fürer and Raghavachari [7]. For the weighted case, Fischer [4] has presented an approximation algorithm that computes a minimum spanning tree of degree at most $b \cdot \Delta^{*}+\left\lceil\log _{b} n\right\rceil$ in time $O\left(n^{4+1 / \ln b}\right)$ for any $b>1$, which is the best-known algorithm for this problem up to now. His algorithm is an adaptation of a local search algorithm of Fürer and Raghavachari [6] to the weighted case. The idea of the local search is to perform edge exchanges until the spanning tree is locally optimal. The crucial point for bounding the runtime is the number of improvements that have to be executed until the approximation guarantee can be given. Fischer has shown, by a similar potential function argument as Fürer and Raghavachari [6], that the number of improvements is bounded by $O\left(n^{2+1 / \ln b}\right)$.

Könemann and Ravi [10] have considered the problem of approximating minimum spanning trees with nonuniform degree bounds. Given degree bounds $B_{v}$ for all vertices, they have presented a combination of the primal-dual method and local search to compute a spanning tree in which the degree of each vertex $v$ is $O\left(B_{v}+\log n\right)$ and the weight is by at most a constant factor higher than the weight of any spanning tree that obeys the given degree constraints. Their algorithm runs in time $O\left(n^{6} \log n\right)$ and the analysis also uses the potential function argument given in [6].

We model the problem of computing minimum spanning forests of minimum degree as a multi-objective optimization problem where one objective is to minimize the number of connected components and the other objective to minimize the weight and degree. Our aim is to compute for each $i, 1 \leq i \leq n$, a minimum spanning forest with $i$ connected components that has the same approximation quality as the algorithm of Fischer. We show that the total number of local improvements can be bounded by $O\left(n^{1+1 / \ln b}\right)$, which is by a factor of $n$ smaller than in the algorithm of Fischer. Based on this analysis we are able to construct a deterministic algorithm that computes a set of solutions containing a minimum 
spanning forest for each number of connected components with the same approximation guarantee as provided by Fischer's algorithm in time $O\left(n^{3+1 / \ln b}\right)$. Our algorithm can be seen as extension of Kruskal's algorithm for the computation of minimum spanning trees. Note that during the run, Kruskal's algorithm computes solutions that are minimum spanning forests for each possible number of connected components. The working principle of our algorithm is also to start with an empty graph and to compute the minimum spanning forests as in the run of Kruskal's algorithm one after another. After a new edge has been introduced that leads to a minimum spanning forest with smaller number of connected components, the degree of this minimum spanning forest is improved by edge exchanges as long as we cannot guarantee our desired approximation quality.

In the case of computing minimum spanning forests obeying given degree bounds we take a similar view. Starting with the empty edge set, we compute in time $O\left(n^{3+2 / \ln b}\right)$ for each number of connected components a minimum spanning forest in which each vertex degree is $O\left(B_{v} \log n\right)$ for any constant $b>1$. Setting $b=e^{k}$ and $k>2 / 3$, this improves the runtime bound given in [10] for the computation of minimum spanning trees with nonuniform degree bounds by a factor $n^{3-2 / k} \log n$. Our algorithm uses an extension of the primal-dual approach given in [10] and our multi-objective view on the problem, which leads to a better bound on the number of local improvements.

The paper is organized as follows. In Section 2, we introduce our model for the computation of minimum spanning forests with minimum degree and give a new algorithm for minimizing the degree of minimum spanning forests that runs in time $O\left(n^{3+1 / \ln b}\right)$. Section 3 applies our technique in combination with an extension of the primal-dual method for minimum spanning trees [10] to the problem of computing minimum spanning forests with nonuniform degree bounds. We finish with some conclusions.

\section{Minimizing the Degree of Minimum Spanning Forests}

\subsection{A Multi-objective Formulation}

We take a multi-objective view on the computation of minimum spanning forests with minimum degree. Let $X=\{0,1\}^{m}$ be the search space. A search point $x \in$ $X$ describes the set of all edges $e_{i}$ where $x_{i}=1$ holds. Let $c(x)$ be the number of connected components of the solution $x, w(x)$ be the weight of the chosen edges, $d_{j}(x)$ be the number of vertices of degree $j$ in $x$, and $\Delta(x)$ the maximum vertex degree of $x$. The value of $x$ is given by the vector $f(x)=\left(f_{1}(x), f_{2}(x)\right)$, where $f_{1}(x)=c(x)$ and $f_{2}(x)=\left(w(x), d_{n-1}(x), \ldots, d_{0}(x)\right)$. Both objectives $f_{1}$ and $f_{2}$ have to be minimized. Minimizing the second objective means minimization with respect to the lexicographic order. This model generalizes the function $g(x)=$ $(c(x), w(x))$ that has been examined by Neumann and Wegener [11] for the computation of minimum spanning trees by randomized search heuristics. They have shown that a multi-objective view leads to a more efficient optimization process than in the case of a single objective one. 
Let $f(X)$ be the image of the search space under the objective function $f$ defined above. By intersecting the canonic order on $f_{1}(X)$ with the lexicographic order on $f_{2}(X)$, both of which are total orders, a partial order on $f(X)$ can be defined as

$$
f(x) \preceq f\left(x^{\prime}\right): \Leftrightarrow f_{1}(x) \leq f_{1}\left(x^{\prime}\right) \wedge f_{2}(x) \leq_{\operatorname{lex}} f_{2}\left(x^{\prime}\right)
$$

for all $x, x^{\prime} \in X$. This partial order represents our preference relation regarding the solutions. The aim is to identify all minimal elements of $(f(X), \preceq)$, and with each minimal element one of its pre-images from $X$.

As the edge weights are positive, a minimum spanning forest with $i$ connected components has a smaller weight than a minimum spanning forest with $i+1$ connected components. Therefore, $(f(X), \preceq)$ has $n$ minimal elements, representing for each $i, 1 \leq i \leq n$, a minimum spanning forest with $i$ connected components and minimum degree. Our goal is to approximate the set of minimal elements as good as possible. We want to compute for each $i$ a minimum spanning forest with $i$ connected components that has degree at most $b \cdot \Delta_{i}^{*}+\left\lceil\log _{b} n\right\rceil$, where $\Delta_{i}^{*}$ is the smallest maximum degree of any minimum spanning forest with $i$ connected components.

\section{$2.2 \quad$ Local Improvements}

Fischer's algorithm [4] for the computation of a minimum spanning tree with degree at most $b \cdot \Delta^{*}+\left\lceil\log _{b} n\right\rceil$ starts with an arbitrary minimum spanning tree and improves the degree of high-degree vertices. The number of these improvements is bounded by $O\left(n^{2+1 / \ln b}\right)$. A better bound on the number of necessary improvements would yield a better upper bound for the runtime of Fischer's algorithm. We consider the number of necessary improvements for our multiobjective model and start with some general properties of minimum spanning forests with $i$ connected components.

Lemma 1. Let $\Delta_{i}^{*}, 1 \leq i \leq n$, be the minimum degree of a minimum spanning forest with $i$ connected components. Then $\Delta_{n}^{*} \leq \Delta_{n-1}^{*} \leq \ldots \leq \Delta_{1}^{*}$ holds.

Proof. Suppose that $\Delta_{i}^{*}>\Delta_{i-1}^{*}$ holds for some $i \in\{2, \ldots n\}$. Let $F_{i-1}^{*}$ be a minimum spanning forest with $i-1$ connected components and minimum degree. Then we can delete the heaviest edge from $F_{i-1}^{*}$ to construct a minimum spanning forest with $i$ connected components whose degree is at most $\Delta_{i-1}^{*}$. This contradicts the assumption.

Let $s_{i}$ be a solution with $i$ connected components and minimal weight. We call $s_{i}$ locally optimal if there is no solution $s_{i}^{\prime}$ with $c\left(s_{i}^{\prime}\right)=c\left(s_{i}\right)$ and hamming distance 2 that is better than $s_{i}$ with respect to $f_{2}\left(s_{i}\right)$ when disregarding all $d_{j}\left(s_{i}\right)$ with $j<\Delta\left(s_{i}\right)-\left\lceil\log _{b} n\right\rceil$. Otherwise, we say that $s_{i}^{\prime}$ improves $s_{i}$. For the case $i=1$ Fischer has shown in [4] that if there is no improvement for $s_{1}$ then the minimum spanning tree has already degree at most $b \cdot \Delta_{1}^{*}+\left\lceil\log _{b} n\right\rceil$ for any $b>1$. We generalize this approximation guarantee of local optimal minimum spanning trees given by Fischer to locally optimal minimum spanning forests. 
Lemma 2. Let $s_{i}$ be a solution that is locally optimal and $\Delta_{i}$ be its maximum degree. Then $\Delta_{i} \leq b \cdot \Delta_{i}^{*}+\left\lceil\log _{b} n\right\rceil$ holds for any constant $b>1$.

Proof. Consider a locally optimal forest $F$ described by $s_{i}$. Let $U_{i}$ be the set of vertices of degree at least $i$ in $F$. The number of vertices in $U_{i}$ is at most $n$ for each $i$. Hence, the ratio $\frac{\left|U_{i-1}\right|}{\left|U_{i}\right|}$ cannot be greater than $b$ for $\log _{b} n$ consecutive values of $i$.

Consider a $\delta$ with $\Delta_{i}-\left\lceil\log _{b} n\right\rceil \leq \delta \leq \Delta_{i}$ such that $\frac{\left|U_{\delta-1}\right|}{\left|U_{\delta}\right|} \leq b$. Deleting all edges from $F$ that are adjacent to vertices of $U_{\delta}$ yields a forest $F_{\delta}$ with at least $(\delta-1)\left|U_{\delta}\right|+1+i$ connected components. As $F$ is locally optimal, only edges adjacent to vertices of $U_{\delta-1}$ may participate in a minimum spanning forest with $i$ connected components. Hence, there must be at least $(\delta-1)\left|U_{\delta}\right|+1 \geq$ $(\delta-1)\left(\left|U_{\delta-1}\right| / b\right)+1$ edges adjacent to vertices of $U_{\delta-1}$. The average degree of a vertex in $U_{\delta-1}$ is therefore at least $\frac{(\delta-1)\left|U_{\delta-1}\right|+b}{b \cdot\left|U_{\delta-1}\right|}$, which implies $\Delta_{i}^{*}>\frac{\delta-1}{b}$. Using $\delta \geq \Delta_{i}-\left\lceil\log _{b} n\right\rceil$ we get $\Delta_{i} \leq b \cdot \Delta_{i}^{*}+\left\lceil\log _{b} n\right\rceil$.

Lemma 3. The total number of local improvements until a minimum spanning forest of degree at most $b \cdot \Delta_{i}^{*}+\left\lceil\log _{b} n\right\rceil$ has been computed for each $i, 1 \leq i \leq n$, can be bounded by $O\left(n^{1+1 / \ln b}\right)$.

Proof. Consider a situation where a minimum spanning forest with $j$ connected components and degree at most $b \cdot \Delta_{j}^{*}+\left\lceil\log _{b} n\right\rceil$ has been computed for each $j$, $i \leq j \leq n$. We want to show that no more than $3 \cdot(n-i) \cdot \mu$ local improvements are necessary to reach this state, where $\mu=O\left(n^{1 / \ln b}\right)$. Setting $i=1$ then proves the lemma.

Let a potential function be defined as $p(s):=\sum_{j=0}^{\left\lceil\log _{b} n\right\rceil+1} d_{r+j}(s) \cdot e^{j}$, where $r=\max \left\{\Delta\left(s_{i}\right)-\left\lceil\log _{b} n\right\rceil, 0\right\}$. The empty edge set $s_{n}=\emptyset$ is obviously a minimum spanning forest with $n$ components and minimum degree, and no improvements are necessary to reach this. In addition $p\left(s_{n}\right)=0$ holds. For going from $i$ to $i-1$ we introduce into $s_{i}$ a lightest edge $e$ that does not create a cycle. This yields a minimum spanning forest with $i-1$ components, denoted as $s_{i-1}^{\prime}$. Introducing an arbitrary edge into $s_{i}$ increases the potential value $p\left(s_{i}\right)$ by at most $2 e^{\left\lceil\log _{b} n\right\rceil+1} \leq$ $2 e^{2} \cdot e^{\log _{b} n}=2 e^{2} \cdot e^{\ln (n) / \ln (b)}=: \mu$, hence $p\left(s_{i-1}^{\prime}\right)-p\left(s_{i}\right) \leq \mu$, where $\mu=$ $O\left(n^{1 / \ln b}\right)$. Now, $s_{i-1}^{\prime}$ can undergo a number $\nu_{i-1}$ of local improvements to arrive at a new solution $s_{i-1}$ that is locally optimal or satisfies $\Delta\left(s_{i-1}\right)=\Delta\left(s_{i}\right)$, which can be achieved by reducing the whole potential $p\left(s_{i-1}^{\prime}\right)$. Due to Lemma 1 and 2 in both cases the claimed approximation holds.

In a local improvement $d_{k}(s)$ decreases by at least 1 for some $k \geq r+2$. The potential reduces by the smallest amount if $d_{k}(s)$ reduces by one, $d_{k-1}(s)$ increases by three and $d_{k-2}(s)$ decreases by 2 . This means that one local improvement step reduces the potential by at least $e^{2}-3 e+2>1 / 3$, i.e., a constant amount.

Therefore, and because $r$ cannot increase in this process, the relation $p\left(s_{i}\right) \leq$ $p\left(s_{i+1}\right)-\nu_{i+1} / 3+\mu$ holds for each $i, 1 \leq i \leq n-1$. Using this, the potential value of a solution $s_{i}^{\prime}$ that has been created by the introduction of a new edge into $s_{i+1}$ can be bounded with respect to the cumulated number of all $\nu_{j}, i<j \leq n$, 
1. Initialize: let $i:=n, s_{i}:=\emptyset, S:=\left\{s_{i}\right\}$.

2. Create $s_{i-1}$ by introducing into $s_{i}$ the lightest edge that does not create a cycle.

3. Improve the solution $s_{i-1}$ until it is locally optimal or $\Delta\left(s_{i-1}\right)=\Delta\left(s_{i}\right)$ holds.

4. $S:=S \cup\left\{s_{i-1}\right\}$

5. $i:=i-1$

6. If $i>1$ continue at 2 , otherwise output $S$ and stop.

Fig. 1. Minimum Spanning Forest Optimizer (MSFO)

previous improvement steps by $p\left(s_{i}^{\prime}\right) \leq(n-i) \mu-\sum_{j=i+1}^{n} \nu_{j} / 3$. As $\nu_{i} \leq 3 p\left(s_{i}^{\prime}\right)$, $\sum_{j=i}^{n} \nu_{j} \leq 3 \mu(n-i)$.

\subsection{The Algorithm}

The analysis in Section 2.2 has shown that the number of improvements in the multi-objective model can be bounded by $O\left(n^{1+1 / \ln b}\right)$, which is by a factor $n$ smaller than then the number of improvements in the algorithm of Fischer. Based on this observation we give a deterministic algorithm that computes for each $i$ a minimum spanning forest with $i$ connected components and degree at most $b \cdot \Delta_{i}^{*}+\left\lceil\log _{b} n\right\rceil$ in time $O\left(n^{3+1 / \ln b}\right)$ for any $b>1$.

Let $s_{i}$ be a minimum spanning forest of degree at most $b \cdot \Delta_{i}^{*}+\left\lceil\log _{b} n\right\rceil$. Then we can produce a minimum spanning forest $s_{i-1}$ with $i-1$ connected components by introducing a lightest edge that does not create a cycle. If $\Delta\left(s_{i-1}\right)=\Delta\left(s_{i}\right)$ holds, $s_{i-1}$ has the desired approximation quality. Otherwise we have to improve $s_{i-1}$. The pseudo-code of our algorithm called Minimum Spanning Forest Optimizer (MSFO) is given in Figure 1.

MSFO can be seen as a variant of Kruskal's algorithm where after each insertion of an edge the degree of the current solution $s_{i-1}$ is improved as long as we cannot guarantee the desired approximation quality. The algorithm of Kruskal can be implemented in time $O((m+n) \log n)$. Hence, to bound the runtime of MSFO it is necessary to bound the number of local improvements (as done in Section 2.2) and the time to achieve such an improvement.

Lemma 4. Let $s_{i}$ be a solution with $i$ connected components that is not locally optimal. Then an improvement can be found in time $O\left(n^{2}\right)$.

Proof. There are two possibilities to improve a solution $s_{i}$. Let $F$ be the corresponding minimum spanning forest. In the first case, the introduced edge connects two components of $F$ and an edge from the forest has to be removed. In the second case, the improvement is achieved by introducing an edge $e$ that creates a cycle in $F$. Then an edge from this cycle has to be deleted to create a new spanning forest with $i$ connected components.

Let $W_{1}, \ldots, W_{k}$ be the distinct weight classes for which there are edges in $F$. For the first case, consider the edges of $W_{j}$ for each $j, 1 \leq j \leq k$, in $F$ and 
compute the edge that reduces the value of the potential function of Lemma 3 by the largest amount. This computation can be done in time $O(n)$ for all weight classes because the computation of the reduction for a single edge $e$ can be done in constant time and there are at most $n-2$ edges to consider. Let $g_{\min }^{j}$ be the smallest value that can be obtained by removing an edge $e_{j}$ of weight class $W_{j}$ from $F$. Now we consider each edge $e^{\prime} \in E \backslash F$ of weight class $W_{j}$ and introduce $e^{\prime}$ if the result improves $s_{i}$. We consider in the process each edge at most once and the computation of the desired potential difference can be implemented in constant time. Hence, an improvement can be found in time $O\left(n^{2}\right)$ in this case.

For the second case we use the idea of Fischer and investigate a depth first search traversal of the forest $F$ represented by $s_{i}$ from every vertex $v \in V$. Let $w$ be the current vertex of the traversal and $P_{w}$ be the set of edges on the path from $v$ to $w$. We assign variables $M_{1}, \ldots, M_{k}$ such that $M_{j}, 1 \leq j \leq k$, denotes the maximum degree of those vertices adjacent to edges of weight class $W_{j}$ in $P_{w}$. For a depth first search traversal we can compute the $M_{i}$ variables in constant time per step using stacks. If there is an edge $(v, w) \in E$, let $w_{i}$ be its weight. If $M_{i}$ is at least two greater than the degree of $v$ and $w$, and $M_{i}$ is at least $\Delta\left(s_{i}\right)-\left\lceil\log _{b} n\right\rceil$, then adding $(v, w)$ to $s_{i}$ and deleting some edge from $P_{w}$ of weight class $W_{i}$ adjacent to a vertex of degree $M_{i}$ constitutes an improvement. The computation of the $n$ depth first search traversals can be carried out in time $O\left(n^{2}\right)$ which completes the proof.

Using the bound on the number of necessary improvements and the time bound to achieve such an improvement, we can give an upper bound on the runtime of MSFO.

Theorem 1. The algorithm MSFO computes for any $b>1$ in time $O\left(n^{3+1 / \ln b}\right)$ a set of solutions that includes for each $i, 1 \leq i \leq n$, a minimum spanning forest with $i$ connected components and degree at most $b \cdot \Delta_{i}^{*}+\left\lceil\log _{b} n\right\rceil$.

Proof. Consider the time the solutions $\left\{s_{n}, s_{n-1}, \ldots, s_{i}\right\} \subset S$ have been produced. These solutions have the following properties. Each $s_{j}, i \leq j \leq n$, is a minimum spanning forest with $j$ connected components. In addition, $s_{j}$ is locally optimal or $\Delta\left(s_{j}\right)=\Delta\left(s_{j+1}\right)$ holds. Obviously, $s_{n}$ is a locally optimal solution. We introduce into $s_{i}$ an edge $e$ of minimal weight that does not create a cycle. This can be easily done by checking each remaining edge in time $O(m)$. Note that the whole computation in step 2 in the run of the algorithm can be implemented in time $O((m+n) \log n)$ using the ideas of Kruskal's algorithm. After step 3, the solution $s_{i-1}$ has minimal weight among all solutions with $i-1$ components. If $e$ is not incident to at least one edge of degree $\Delta\left(s_{i}\right), \Delta\left(s_{i-1}\right)=\Delta\left(s_{i}\right)$ holds. Otherwise, the number of vertices with degree $\Delta\left(s_{i}\right)+1$ is at most 2 and we have to improve $s_{i-1}$ to reach a locally optimal solution or to achieve $\Delta\left(s_{i-1}\right)=\Delta\left(s_{i}\right)$.

The number of local improvements in the run of MSFO is $O\left(n^{1+1 / \ln b}\right)$ as shown in Lemma 3 and an improvement of a non locally optimal solution can be found in time $O\left(n^{2}\right)$ due to Lemma 4. Hence, the time until MSFO has achieved the desired approximation can be bounded by $O\left(n^{3+1 / \ln b}\right)$. 


\section{Minimum Spanning Forests with Nonuniform Degree Bounds}

Könemann and Ravi [10] have examined the case of non-uniform degree bounds $B_{v}$ for all vertices $v \in V$. They presented an algorithm that finds, in time $O\left(n^{6} \log n\right)$, a spanning tree where the degree of each vertex is $O\left(B_{v}+\log n\right)$ and whose total edge weight is at most a constant times the weight of any tree that satisfies the degree constraints. The algorithm uses a combination of primaldual methods and local search, where in each local search step the normalized degree of the high-degree vertices in a current spanning tree is reduced. We generalize the primal-dual idea of Könemann and Ravi to the approximation of minimum spanning forests with nonuniform degree bounds. The task is to find for each $i, 1 \leq i \leq n$, a spanning forest with $i$ connected components and minimum total edge weight such that the maximum degree of each vertex $v$ is at most $B_{v}$. The algorithm presented here runs in time $O\left(n^{3+2 / \ln b}\right), b>1$ an arbitrary constant, and outputs for each $i, 1 \leq i \leq n$, a spanning forest $F_{i}$ of $i$ connected components whose vertex degrees are $O\left(B_{v}+\log n\right)$ and whose total weight is at most a constant times the total weight of any minimum spanning forest with $i$ connected components.

We first adapt some results of [10] to the case of spanning forests. A feasible partition of $V$ is a set $\pi=\left\{V_{1}, \ldots, V_{k}\right\}$ where $V_{i} \cap V_{j}=\emptyset$ for all $i \neq j, V=$ $V_{1} \cup \ldots \cup V_{k}$, and the induced subgraphs $G\left[V_{i}\right]$ are connected. Let $G_{\pi}$ be the graph obtained from $G$ by contracting each $V_{i}$ into a single vertex, $\Pi$ be the set of all feasible partitions of $V$, and $x(e)$ be the variable indicating whether edge $e$ is included in the current solution, i.e., $x\left(e_{i}\right)=x_{i}$. We consider the following integer linear program (IP) formulation for the problem of computing the minimum spanning forest with $i, 1 \leq i \leq n$, connected components that obeys all degree bounds $B_{v}$.

$$
\begin{array}{ll}
\min & \sum_{e \in E} w(e) x(e) \\
\text { s.t. } & \sum_{e \in E\left[G_{\pi}\right]} x(e) \geq|\pi|-i \quad \forall \pi \in \Pi \\
& \sum_{e \in E: v \in e} x(e) \leq B_{v} \quad \forall v \in V \\
& x(e) \in\{0,1\} \quad \forall e \in E
\end{array}
$$

The dual of the linear programming relaxation (LP) of (IP) is given by

$$
\begin{array}{ll}
\max & \sum_{\pi \in \Pi}(|\pi|-i) \cdot y_{\pi}-\sum_{v \in V} \lambda_{v} B_{v} \\
\text { s.t. } & \sum_{\pi: e \in E\left[G_{\pi}\right]} y_{\pi} \leq w(e)+\lambda_{u}+\lambda_{v} \quad \forall e=(u, v) \in E \\
& y, \lambda \geq 0
\end{array}
$$


Könemann and Ravi have given a primal-dual interpretation of Kruskal's algorithm. Let (IP-SP) denote (IP) without constraints of type (3) its LP relaxation denoted by (LP-SP) and its dual be (D-SP). Kruskal's algorithm can be seen as a continuous process over time that starts with an empty edge set at time 0 and ends with a minimum spanning tree at time $t^{*}$. At any time $t, 0 \leq t \leq t^{*}$, a pair $\left(x^{t}, y^{t}\right)$ is kept, where $x^{t}$ is a partial primal solution for (LP-SP) and $y^{t}$ is feasible solution for (D-SP). In the initialization step $x(e)^{0}=0$ is set for all $e \in E$, and $y_{\pi}^{t}=0$ for all $\pi \in \Pi$. Consider the forest $F^{t}$ that corresponds to the partial solution $x^{t}$ and let $\pi^{t}$ be the partition induced by the connected components of $G\left[F^{t}\right]$. At time $t$ the algorithm increases $y_{\pi}^{t}$ until a constraint of type (6) becomes tight. If this happens for edge $e$, this edge $e$ is included into the primal solution. If more than one edge becomes tight, the edges are processed in arbitrary order. We denote by $\mathrm{MSF}_{i}$ a variant of this algorithm that stops when a minimum spanning forest with $i$ connected components has been computed in the continuous process.

Let $\operatorname{deg}_{F}(v)$ be the degree of vertex $v$ in the spanning forest $F$ with $i$ connected components. The normalized degree of a vertex $v$ is denoted by $n \operatorname{deg}_{F}(v)=\max \left\{0, \operatorname{deg}_{F}(v)-1-b \alpha \cdot B_{v}\right\}$, where $b$ and $\alpha$ are constants depending on the desired approximation quality. Let $\Delta^{t}$ the maximum normalized degree of any vertex in the current spanning forest $F_{i}^{t}$ at a given time $t$ and denote by $U_{j}^{t}$ the set of vertices whose normalized degree is at least $j$ at time $t$. The following lemma was shown in [10].

Lemma 5. There is a $d^{t} \in\left\{\Delta^{t}-2 \log _{b} n, \ldots, \Delta^{t}\right\}$ such that

$$
\sum_{v \in U_{d^{t}}-1} B_{v} \leq b \cdot \sum_{v \in U_{d^{t}}} B_{v}
$$

for any constant $b>1$.

Proof. Suppose that for all $d^{t} \in\left\{\Delta^{t}-2 \log _{b} n, \ldots, \Delta^{t}\right\}$ the relation

$$
\sum_{v \in U_{d^{t}}-1} B_{v}>b \cdot \sum_{v \in U_{d^{t}}} B_{v}
$$

holds. We may assume $B_{v} \leq n-1$ for any $v$, which implies $\sum_{v \in V} B_{v} \leq n(n-1)$. Since there is at least one vertex of normalized degree $\Delta^{t}$, we have

$$
\sum_{v \in U_{\Delta^{t}-2 \log _{b} n}} B_{v} \geq b^{2 \log _{b} n}=n^{2},
$$

a contradiction.

Our algorithm called Primal Dual Forest Optimizer (PDFO) is given in Figure 2. The idea of the algorithm is to start with an empty edge set and compute the solutions with the desired approximation quality one after another. If we are 
1. $t:=0 ; \lambda_{v}^{t}:=0, \forall v \in V ; w^{t}(e)=w(e), \forall e \in E$

2. $i:=n ;\left(x^{t}, y^{t}\right):=\operatorname{MSF}_{i}\left(G, w^{t}\right) ; S:=\left\{x^{t}\right\}$;

3. while $i>1$ do

(a) $i:=i-1 ; w^{t+1}(e)=w^{t}(e) ;\left(x^{t+1}, y^{t+1}\right):=\operatorname{MSF}_{i}\left(G, w^{t+1}\right) ; t:=t+1$;

(b) while $\Delta^{t}>2 \log _{b} n$ do

i. Choose $d^{t} \in\left\{\Delta^{t}-2 \log _{b} n, \ldots, \Delta^{t}\right\}$ s.t. $\sum_{v \in U_{d^{t}-1}} B_{v} \leq b \cdot \sum_{v \in U_{d^{t}}} B_{v}$

ii. Choose $\epsilon^{t}$ and let $\lambda_{v}^{t+1}:=\lambda_{v}^{t}+\epsilon^{t}$ if $v \in U_{d^{t}-1}^{t}$ and $\lambda_{v}^{t+1}:=\lambda_{v}^{t}$ otherwise

iii. $w^{t+1}(e):=w^{t}(s)+\epsilon^{t}$ if $\left(\left(e \in F_{i}^{t}\right) \wedge\left(e \cap U_{d^{t}} \neq \emptyset\right) \vee\left(\left(e \notin F_{i}^{t}\right) \wedge\left(e \cap U_{d^{t}-1} \neq \emptyset\right)\right)\right.$ and $w^{t+1}(e):=w^{t}(e)$ otherwise

iv. $\left(x^{t+1}, y^{t+1}\right):=\operatorname{MSF}_{i}\left(G, w^{t+1}\right) ; t:=t+1$;

(c) $S:=S \cup\left\{x^{t}\right\}$;

Fig. 2. Primal Dual Forest Optimizer (PDFO)

considering a solution $x^{t}$ with $i$ connected components that does not have the desired approximation quality with respect to the degree bounds, we compute a new solution $x^{t+1}$ which improves $x^{t}$ with respect to the normalized degree. Let $F_{i}^{t}$ be the forest corresponding to $x^{t}$. We increase the weight of an edge $e \in E$ by $\epsilon^{t}$ if it is either in $F_{i}^{t}$ and adjacent to vertices of $U_{d^{t}}$, or in $E \backslash F_{i}^{t}$ and adjacent to vertices of $U_{d^{t}-1}$. The weight increment $\epsilon^{t}$ is defined as the smallest weight increase when deleting an edge adjacent to a vertex of $U_{d^{t}}$ and inserting an edge adjacent to vertices that are not contained in $U_{d^{t}-1}$ such that a new cyclefree subgraph of $G$ is constructed. After that, $x^{t+1}$ is a minimum spanning forest with $i$ connected components with respect to the updated weight function $w^{t+1}$. We have also stated the computation of the dual variables corresponding to the primal solutions in Figure 2 using the algorithm $\mathrm{MSF}_{\mathrm{i}}$. The dual variables will be used later to show the approximation quality of our algorithm, but it is not necessary to carry out the computation of these variables in the run of the algorithm.

We want to show that the algorithm computes in time $O\left(n^{3+2 / \ln b}\right)$ a solution set that contains for each $i, 1 \leq i \leq n$, a spanning forest with $i$ connected components in which each vertex $v$ has degree $O\left(B_{v}+\log n\right)$ and weight at most a constant times the weight of an optimal solution obeying the degree bounds. First, we consider the approximation quality of the solutions that are introduced into the set $S$ in step 3c. Here we use an extension of the arguments given in [10] to the case of minimum spanning forest with given degree bounds.

Lemma 6. For all iterations $t \geq 0$ where we are considering solutions with $i$ connected components in the algorithm PDFO, the relation

$$
\sum_{\pi \in \Pi}(|\pi|-j) y_{\pi}^{t+1} \geq \sum_{\pi \in \Pi}(|\pi|-j) y_{\pi}^{t}+\epsilon^{t} \alpha \sum_{v \in U_{d^{t}-1}} B_{v}
$$

holds for and all $j, 1 \leq j \leq i$.

Proof. We generalize the ideas in the proof of Claim 1 in [10]. Let $F_{j}^{t}=\left\{e_{1}^{t}, \ldots, e_{n-j}^{t}\right\}$ be the set of edges that would be produced by a run of the algorithm $\mathrm{MSF}_{j}$ in 
iteration $t$. The change of the dual objective function value in iteration $t$ for a specific value of $j$ is given by

$$
\sum_{\pi \in \Pi}(|\pi|-j) \cdot\left(y_{\pi}^{t+1}-y_{\pi}^{t}\right)=\sum_{l=1}^{n-j}\left(r_{l}^{t+1}-r_{l}^{t}\right)
$$

where $r_{l}^{t}$ is the time at which the $\mathrm{MSF}_{j}$ algorithm includes the edge $e_{l}^{t}$. Assume that we are considering solutions with $i$ connected components in iteration $t$. Then we lengthen all edges $e \in F_{i}^{t}$ that are incident to vertices of normalized degree at least $d^{t}$. This implies that all these edges become tight $\epsilon^{t}$ time later. Using that all edges of $F_{i}^{t}$ are also contained in each minimum spanning forest $F_{j}^{t}$ for $j \leq i$, we get

$$
\sum_{\pi \in \Pi}(|\pi|-j) \cdot\left(y_{\pi}^{t+1}-y_{\pi}^{t}\right) \geq \epsilon^{t} \cdot\left|E\left(U_{d^{t}}\right) \cap F_{i}^{t}\right| .
$$

Here $E\left(U_{d^{t}}\right)$ denotes the set of edges that are incident to vertices from $U_{d^{t}} . F_{i}^{t}$ is a minimum spanning forest with $i$ connected components. This implies that there are at most $\left|U_{d^{t}}\right|-i$ edges in $E\left(U_{d^{t}}\right)$ that are incident to two vertices from $U_{d^{t}}$, therefore

$$
\epsilon^{t} \cdot\left|E\left(U_{d^{t}}\right) \cap F_{i}^{t}\right| \geq \epsilon^{t} \cdot\left[\left(\sum_{v \in U_{d^{t}}}\left(b \alpha+1 / B_{v}\right) \cdot B_{v}\right)-\left(\left|U_{d^{t}}\right|-i\right)\right] .
$$

This leads to

$$
\sum_{\pi \in \Pi}(|\pi|-j)\left(y_{\pi}^{t+1}-y_{\pi}^{t}\right) \geq \epsilon^{t} \alpha b \cdot\left(\sum_{v \in U_{d^{t}}} B_{v}\right)+i \geq \epsilon^{t} \alpha b \cdot \sum_{v \in U_{d^{t}}} B_{v},
$$

and using Lemma 5 we get

$$
\sum_{\pi \in \Pi}(|\pi|-j)\left(y_{\pi}^{t+1}-y_{\pi}^{t}\right) \geq \epsilon^{t} \alpha \cdot \sum_{v \in U_{d^{t}-1}} B_{v},
$$

which completes the proof.

Lemma 7. Let $\omega>1$ be a constant and $\alpha=\max \{\omega /(\omega-1), \omega\}$. For all iterations $t \geq 0$ where we are considering solutions with $i$ connected components in the algorithm PDFO, the relation

$$
\omega \sum_{v \in V} B_{v} \lambda_{v}^{t} \leq(\omega-1) \sum_{\pi \in \Pi}(|\pi|-j) \cdot y_{\pi}^{t}
$$

holds for each $j, 1 \leq j \leq i$.

Proof. After initialization, $\sum_{v \in V} B_{v} \lambda_{v}^{0}=\sum_{\pi \in \Pi}(|\pi|-j) \cdot y_{\pi}^{0}=0$ holds. Lemma 6 implies that the right hand side of (9) increases by at least $(\omega-1) \cdot \alpha \epsilon^{t} \sum_{v \in U_{d^{t}-1}^{t}} B_{v}$. The left hand side increases by $\omega \cdot \epsilon^{t} \sum_{v \in U_{d^{t}-1}^{t}} B_{v}$. Using $\alpha \geq \omega /(\omega-1)$, the relation is maintained. 
Lemma 8. For all iterations $t \geq 0$ where we are considering solutions with $i$ connected components in the algorithm PDFO, the relation

$$
\sum_{e \in F_{j}^{t}} w(e) \leq \omega\left[\sum_{\pi \in \Pi}\left((|\pi|-j) \cdot y_{\pi}^{t}\right)-\sum_{v \in V}\left(B_{v} \cdot \lambda_{v}^{t}\right)\right]
$$

holds for each $j, 1 \leq j \leq i$.

Proof. For $t=0$ this is obviously true. Let $F_{j}^{t}$ be the spanning forest produced by the algorithm $\mathrm{MSF}_{j}$ in the $t$-th iteration and let $w^{t}\left(F_{j}^{t}\right)$ be the weight of this spanning forest with respect to the weight function $w^{t}$. As the weights can only increase during the run of PDFO,

$$
w\left(F_{j}^{t}\right) \leq w^{t}\left(F_{j}^{t}\right)=\sum_{e \in F_{j}^{t}} w^{t}(e)=\sum_{\pi \in \Pi}(|\pi|-j) \cdot y_{\pi}^{t}
$$

holds. Using the invariant given in Lemma 7 we get

$$
w\left(F_{j}^{t}\right) \leq \omega\left[\sum_{\pi \in \Pi}\left((|\pi|-j) \cdot y_{\pi}^{t}\right)-\sum_{v \in V}\left(B_{v} \cdot \lambda_{v}^{t}\right)\right]
$$

Lemma 8 shows that in each iteration the weight of a spanning forest with $j$, $1 \leq j \leq i$, is only a constant times the weight of an optimal solution. It remains to show an upper bound on the runtime of PDFO. To do this we first consider the time to produce a new solution $x^{t+1}$ from the current solution $x^{t}$.

Lemma 9. The solution $x^{t+1}$ can be computed from $x^{t}$ in time $O\left(n^{2}\right)$.

Proof. If the computation of $x^{t+1}$ is carried out in step 3a of the algorithm introducing the lightest edge for the weight function $w^{t+1}$ into $x^{t}$ that does not create a cycle yields $x^{t+1}$. This can be done in time $O\left(n^{2}\right)$ by inspecting every edge at most once. In the other case $x^{t}$ is a minimum spanning forest with $i$ connected components with respect to $w^{t}$ and $x^{t+1}$ is a minimum spanning forest with $i$ connected components with respect to the updated weight function $w^{t+1}$. To determine $x^{t+1}$ we have to compute $\epsilon^{t}$ and execute the resulting exchange operation. For the computation of $d^{t}$ we use an integer array of size $n$ and store at position $j, 0 \leq j \leq n-1$, the sum over the $B_{v}$-values with vertices of normalized degree $j$ in $F_{i}^{t}$. This can be done in time $O(n)$ using a breath first search traversal on $F_{i}^{t}$ in which we compute the $B_{v}$ value for the current vertex $v$ in the traversal and add the value to the value of the corresponding position in the array. After that we determine the values $\sum_{v \in U_{j}} B_{v}, 0 \leq j \leq n-1$, one after another starting with $U_{0}$. Note that $\sum_{v \in U_{0}} B_{v}=\sum_{v \in V} B_{v}$. The value $\sum_{v \in U_{j+1}} B_{v}$ can be computed by subtracting from $\sum_{v \in U_{j}} B_{v}$ the entry at position $j$ in the array. Each computation can be done in constant time based on the corresponding array values. Hence, the value $d^{t}$ due to Lemma 5 can be determined in time 
$O(n)$. To compute the $\epsilon^{t}$ value we determine the exchange operation that leads to a primal solution of $\operatorname{MSF}_{i}\left(G, w^{t+1}\right)$. Note that $\epsilon^{t}$ is the smallest weight increase such that deleting an edge adjacent to at least one vertex of $U_{d^{t}}$ and inserting an edge adjacent to vertices that are not contained in $U_{d^{t}-1}$ yields a minimum spanning forest with $i$ components for the weight function $w^{t+1}$. We consider two possibilities for the exchange operation.

First we investigate the case where introducing an edge $e$ connects two components of the current forest $F_{i}^{t}$. Then another edge from the resulting forest has to be removed to create a solution with $i$ connected components. Introducing the edge $e$ with smallest weight that is not incident to vertices of $U_{d^{t}-1}$ and deleting the edge $e^{\prime} \in F_{i}^{t}$ that has the largest weight of all edges incident to vertices of $U_{d^{t}}$ in $F_{i}^{t}$ leads to the desired exchange operation with the smallest weight increase. Each edge of $G$ has to be examined once, which gives an upper bound of $O\left(n^{2}\right)$ on the runtime in this case. Let $\epsilon^{t^{\prime}}$ be the value obtained by this exchange operation.

The other possibility to get a smaller value than $\epsilon^{t^{\prime}}$ is to introduce into $F_{i}^{t}$ an edge that creates a cycle. Then we have to delete one edge of this cycle. We use a depth first search traversal of $F_{i}^{t}$ from every vertex $v \in V$. Let $w$ be the current vertex in this traversal. Assume that there is an edge $e=(v, w)$ in $E$ and that $v$ and $w$ are not contained in $U_{d^{t}-1}$. Otherwise we can continue the traversal since the pair $(v, w)$ does not fulfill the properties for the exchange operation. Let $w_{i}$ be the largest weight of an edge $e^{\prime}$ in the path from $v$ to $w$ that is incident to vertices of $U_{d^{t}}$. If no such edge edge $e^{\prime}$ exists in the path from $v$ to $w, e$ can not participate in the exchange operation we are looking for. The weight increase of introducing $e$ and deleting $e^{\prime}$ can be computed in constant time, and the $w_{i}$ variables can be maintained in constant time per step of the traversal using stacks. Hence, we can determine the exchange operation with the smallest weight increase in the second case in time $O\left(n^{2}\right)$. Let $\epsilon^{t^{\prime \prime}}$ be weight increase of this exchange operation. Choosing $\epsilon^{t}=\min \left\{\epsilon^{t^{\prime}}, \epsilon^{t^{\prime \prime}}\right\}$ and computing a primal solution of $\mathrm{MSF}_{i}^{t+1}$ by executing the corresponding exchange operation gives the stated upper bound. In addition we update the weight with respect to $w^{t+1}$ for the next iteration which can be done in time $O\left(n^{2}\right)$.

Theorem 2. The algorithm PDFO computes for any $b>1$ and $\omega>1$ in time $O\left(n^{3+2 / \ln b}\right)$ a set of solutions that includes for each $i, 1 \leq i \leq n$, a minimum spanning forest with $i$ connected components in which each vertex degree is at most $b \cdot \alpha \cdot B_{v}+2 \log _{b} n+1$ and the total weight is at most $\omega \cdot w\left(F_{i}^{*}\right)$, where $\alpha=\max \{\omega /(\omega-1), \omega\}$ and $w\left(F_{i}^{*}\right)$ is the minimum weight of any spanning forest with $i$ connected components satisfying the degree bounds.

Proof. As long as the algorithm has not achieved a solution with $i$ connected components such that the vertex degree is at most $b \cdot \alpha \cdot B_{v}+2 \log _{b} n+1$, the right hand side of (10) is $\omega$ times the optimal value of the dual objective function. This implies that the weight of a minimum spanning forest with $i$ connected components for the weight function $w^{t}$ is at most $\omega$ times the value of an optimal solution obeying the degree bounds for each $j, 1 \leq j \leq i$. Hence, the solutions 
introduced into the set $S$ in step 3c of PDFO have the stated approximation quality. As each possible value of $i$ is considered in the run of PDFO, the set $S$ includes after termination for each $i, 1 \leq i \leq n$, a solution with $i$ connected components that has the desired approximation quality.

In the following we give an upper bound of $O\left(n^{3+2 / \ln b}\right)$ on the runtime until the algorithm terminates. A new solution $x^{t+1}$ can be computed from the current solution in time $O\left(n^{2}\right)$ due to Lemma 9. It remains to bound the number of primal solutions $x^{t}$ that have to be computed until the algorithm terminates. The number of solutions that are computed in step 3a of the algorithm is at most $n-1$ as the number of connected components is bound by $n$. In the inner while-loop we compute for the solution with $i$ connected components new primal solutions as long as we have not reached a solution with $i$ connected components that has the desired approximation quality.

We consider a modification of the potential function $p$ introduced in Lemma 3. Consider a solution $s$, let $\hat{\Delta}(s)$ be the maximum normalized degree of $s$, and let $\hat{d}_{i}, 0 \leq i \leq n-1$, be the number of vertices with normalized degree $i$ in this solution. The potential of a solution $s$ is given by $p^{\prime}(s):=\sum_{j=0}^{\left\lceil 2 \log _{b} n\right\rceil+1} \hat{d}_{r+j}(s)$. $e^{j}$, where $r=\hat{\Delta}(s)-\left\lceil 2 \log _{b} n\right\rceil$. Assume the a minimum spanning forest with $i, 2 \leq i \leq n$, has been computed. After initialization this is true for $i=n$. The solution of $\operatorname{MSF}_{i-1}\left(G, w^{t}\right)$ differs from $\operatorname{MSF}_{i}\left(G, w^{t}\right)$ by one single edge that is additionally introduced into $\operatorname{MSF}_{i-1}\left(G, w^{t}\right)$ at any time $t$. Introducing this edge into $s_{i}$, a solution $s_{i-1}^{\prime}$ with $p^{\prime}\left(s_{i-1}^{\prime}\right)-p^{\prime}\left(s_{i}\right)=O\left(n^{2 / \ln b}\right)$ is created. Each iteration of the inner while-loop reduces the potential by at least $1 / 3$. Hence, we can upper bound the number of improvements in the run of PDFO by $O\left(n^{1+2 / \ln b}\right)$ using the ideas of Lemma 3 .

Note that choosing $b$ as a constant large enough the runtime of PDFO approximates the upper bound $O\left(n^{3}\right)$. For $b=e, b=e^{2}, \ldots, b=e^{k}$, where $e=2.71 \ldots$ and $k$ is a constant, we get runtimes $O\left(n^{5}\right), O\left(n^{4}\right), \ldots, O\left(n^{3+2 / k}\right)$. The degrees of the produced solutions are bounded by $O\left(B_{v} \log n\right)$, and the weight of a solution with $i$ connected components introduced into the set $S$ in step 3c is at most a constant times the weight of any minimum spanning forest with $i$ connected components obeying the degree bounds.

\section{Conclusions}

We have given faster approximation algorithms for the generalization of two NPhard spanning tree problems. These algorithms are based on the multi-objective view which enables use to reduce the number of necessary improvements until the approximation guarantee can be given. Based on these observations we have given an algorithm that computes for each $i, 1 \leq i \leq n$, a minimum spanning forest with $i$ components and degree at most $b \cdot \Delta_{i}^{*}+\left\lceil\log _{b} n\right\rceil$ in a total time of $O\left(n^{3+1 / \ln b}\right)$. In the case of nonuniform degree bounds we have presented an algorithm that runs in time $O\left(n^{3+2 / \ln b}\right)$ and computes for each $i, 1 \leq i \leq n$, a spanning forest in which each vertex has degree $O\left(B_{v}+\log n\right)$ and the weight 
is a most a constant times the weight of a minimum spanning forest with $i$ components obeying the given degree bounds.

\section{References}

1. Camerini, P. M., Galgiati, G, and Maffioli, F. (1980). Complexity of spanning tree problems. European Journal on Operation Research, 5: 346-252.

2. Caro, Y., Krasikov, I., and Roditty, Y. (1991). On the largest tree of a given maximum degree in a connected graph. Journal of Graph Theory, 15: 7-13.

3. Cormen, T., Leiserson, C., Rivest, R., and Stein, C. (2001). Introduction to Algorithms. 2nd Edition, McGraw Hill, New York.

4. Fischer, T. (1993). Optimizing the Degree of Minimum Weight Spanning Trees. Technical Report 93-1338, Department of Computer Science, Cornell University, Ithaca, NY, USA.

5. Fürer, M., and Raghavachari, B. (1990). An NC approximation algorithm for the minimum degree spanning tree problem. In Proc. of the 28th Annual Allerton Conf. on Communication, Control and Computing, 274-281.

6. Fürer, M., and Raghavachari, B. (1992). Approximating the Minimum-Degree Spanning Tree to within One from the Optimal Degree. In Proc. of the third annual ACM-SIAM symposium on Discrete algorithms (SODA), 317-324.

7. Fürer, M., and Raghavachari, B. (1994). Approximating the Minimum-Degree Steiner Tree to within One of Optimal. Journal of Algorithms 17, 409-423.

8. Garey, M. R., Johnson, D. S. (1979). Computers and Intractability: A Guide to the Theory of NP-completeness. Freeman, New York.

9. Goemans, M. X., and Williamson, D. P. (1995). A general approximation technique for constrained forest problems. SIAM J. Comput. 24, 296-317

10. Könemann, J., and Ravi, R. (2003). Primal-dual meets local search: approximating MST's with nonuniform degree bounds. In Proc, ACM Symposium on Theory of Computing (STOC), 389-395

11. Neumann, F. and Wegener, I. (2005). Minimum spanning trees made easier via multi-objective optimization. Accepted for GECCO 2005. 